Nonlinear Processes in Geophysics, 12, 807-815, 2005

SRef-ID: $1607-7946 / \mathrm{npg} / 2005-12-807$

European Geosciences Union

(C) 2005 Author(s). This work is licensed

under a Creative Commons License.

\title{
Using MSSA to determine explicitly the oscillatory dynamics of weakly nonlinear climate systems
}

\author{
S. Raynaud ${ }^{1}$, P. Yiou ${ }^{1}$, R. Kleeman ${ }^{2}$, and S. Speich ${ }^{3}$ \\ ${ }^{1}$ Laboratoire des Sciences du Climat et de l'Environnement, CEA, Gif-sur-Yvette, France \\ ${ }^{2}$ Courant Institude of Mathematical Sciences, NYU, New-York, USA \\ ${ }^{3}$ Laboratoire de Physique des Océans, UBO, Brest, France
}

Received: 23 March 2005 - Revised: 4 August 2005 - Accepted: 4 August 2005 - Published: 31 August 2005

\begin{abstract}
This paper presents a statistical diagnostic to interpret the dynamics of nonlinear variability in models. It consists in applying the Multichannel Singular Spectrum Analysis to the variability of a suitable equation of a dynamical system. This method enables us to evaluate the sensitivity of the oscillatory features of a physical model to tendencies in its mathematical formulation. Hence, responses in amplitude and frequency are determined in the phase space of the model. The example of interannual variability of the tropical Pacific climate through the analysis the Sea Surface Temperature equation of a hybrid coupled model is used to illustrate and validate the methodology.
\end{abstract}

\section{Introduction}

Statistical techniques have often been used as diagnostics of climate dynamics on observations or simulations (von Storch and Zwiers, 2001; Wilks, 1995). Ghil and Robertson (2000) and Ghil et al. (2002), and others argue that the attractor of climate systems can be decomposed into sets of possibly unstable limit cycles. This approach motivates the computation of the spectral properties of climate variables, or fields of variables, in order to reconstruct the dynamics of the system (Ghil, 2002). The Singular Spectrum Analysis (SSA) has become a standard tool for such analysis (Broomhead and King, 1986; Vautard and Ghil, 1989; Elsner and Tsonis, 1991, 1996; Golyandina et al., 2001). Its multi-variate extension (MSSA), combined with principal component analysis (PCA), allowed Plaut and Vautard (1994) to investigate the spatio-temporal variability of the atmospheric circulation.

SSA is aimed at reconstructing the features of the attractor of an ordinary differential equation (Broomhead and King, 1986). This analysis uses Takens' method of delays to embed the trajectory of the system into an ad hoc phase space. This delay-embedding procedure is very useful in the case

Correspondence to: S. Raynaud

(stephane.raynaud@cea.fr) of low-dimensional systems, typically when their dimension $d \leq 3$. But it is impractical in higher dimensions, which is the case for the climate system. Hence, SSA helps finding the few modes of the underlying dynamics by decomposing their variance onto an orthogonal base of oscillators. Its properties were investigated for random processes (Allen and Smith, 1996) and nonstationnary processes (Yiou et al., 2000), which often occur in geosciences.

Multi-Channel SSA (MSSA) is an extension of SSA to describe the behavior of evolutive partial differential equations. In this case, each spatial dimension is called a "channel". MSSA takes advantage of the delay embedding procedure to obtain a similar formulation as SSA, albeit with larger matrices. MSSA is hence appropriate for the study of climate fields, as demonstrated by Plaut and Vautard (1994).

One of the applications of SSA or MSSA is to project the observed trajectory of the analyzed system onto one of the prominent limit cycles of its attractor. This allows heuristic interpretations of the dynamics of the system. The main caveat of this interpretation is that the observations have to be generated by a deterministic dynamical system, which is a rather strong assumption in the case of some climate observations. Otherwise, SSA can be assimilated as a data adaptive bandpass filter. However, by directly applying the same filter to a closed physical equation, it is possible to achieve physical consistency to the diagnosed variability. Models easily offer closed equations and can be analysed in this way.

The goal of this paper is to apply MSSA to actual equations of climate dynamics in order to derive the oscillatory properties of their solutions. We investigate cases of perturbed system and the impact on the cyclic features of its attractor. We choose the example of the El-Niño - Southern Oscillation (ENSO) phenomenon to illustrate the power of our approach.

Section 2 is devoted to basic formulation of SSA, and its application to an oscillating dynamical system. Section 3 treats an application of this tool to the equations of a simple model of ENSO. Conclusions appear in Sect. 4. 


\section{Methodology}

\subsection{The analysis method}

MSSA combines the classical principal component analysis (PCA, Preisendorfer, 1988; Wilks, 1995; von Storch and Zwiers, 2001) to reduce the phase space dimension, and SSA. This analysis is the multivariate extension of SSA and can also be seen as the temporal extension on the Empirical Orthogonal Functions (EOFs) of PCA (Plaut and Vautard, 1994).

Using the same formalism as Plaut and Vautard (1994), we consider a spatio-temporal field $\left\{\mathbf{X}_{\ell i}\right\}, \ell$ being the spatial index $(1 \leq \ell \leq L)$ and $i$ the discrete temporal index $(1 \leq i \leq N)$.

The principal component (PC) decomposition $\left\{a^{k}\right\}$ of the field $\mathbf{X}_{\ell i}$ on an orthonormal basis of spatial EOFs $\left\{\mathbf{E}^{k}\right\}$ can be written as:

$\mathbf{X}_{\ell i}=\sum_{k=1}^{L} a_{i}^{k} \mathbf{E}_{\ell}^{k}$

In the same way, we can write the SSA expansion of a single channel series $\left\{\mathbf{x}_{i}\right\}$ on the temporal EOFs basis:

$\mathbf{x}_{i+j}=\sum_{k=1}^{M} a_{i}^{k} \mathbf{E}_{j}^{k}$

$M$ being the key parameter (the "window") of the method. Recombining the two equations, we obtain for the MSSA the decomposition on space-time EOFs $\left\{\mathbf{E}_{\ell j}^{k}\right\}(1 \leq j \leq M)$ :

$\mathbf{X}_{\ell i+j}=\sum_{k=1}^{L} a_{i}^{k} \mathbf{E}_{\ell j}^{k}$

The EOFs are the eigenvectors of the block-Toeplitz crosscovariance matrix $\mathbf{T}$ of $\mathbf{X}$ :

$\mathbf{T} \mathbf{E}=\Lambda \mathbf{E}$,

where $\Lambda$ are the eigenvalues of $\mathbf{T}$, and

$\left(\mathbf{T}_{\ell \ell^{\prime}}\right)_{j j^{\prime}}=\frac{1}{N-\left|j-j^{\prime}\right|} \sum_{i=1}^{N-\left|j-j^{\prime}\right|} \mathbf{X}_{\ell i} \mathbf{X}_{\ell^{\prime} i+j-j^{\prime}}$

where $\ell^{\prime}$ and $j^{\prime}$ refer to similar indexes to $\ell$ and $j$ previously defined $\left(\left(\mathbf{T}_{\ell \ell^{\prime}}\right)_{j j^{\prime}}\right.$ is the lag covariance of $\mathbf{X}$ between position $\ell$ and $\ell^{\prime}$, with a lag of $\left.j-j^{\prime}\right)$. Each mode given by the MSSA has specific spatial, spectral, and propagative properties. MSSA has been used for extracting regular oscillations from short noisy time series. Pairs of modes, with similar variance and timescale, have been shown to account for regular oscillatory patterns (Vautard and Ghil, 1989).

This decomposition can be extended to any linear combination of the field $\mathbf{X}_{\ell i}$. Suppose that $\mathcal{C}$ is a linear operator such as $\mathcal{C}_{\ell}\left(\mathbf{X}_{i}\right)=0$ operating on $\ell$ and for each given time $i$. Hence one or more linear dependencies between the channels of $\mathbf{X}$ can be introduced. Applying $\mathcal{C}$ to the eigen Eq. (4) for the $k^{\text {th }}$ component and using Eqs. (3) and (5) gives:

$$
\begin{gathered}
\sum_{j^{\prime}=1}^{M} \frac{1}{N-\left|j-j^{\prime}\right|} \sum_{l^{\prime}=1}^{L} \sum_{i=1}^{N-\left|j-j^{\prime}\right|} \mathbf{E}_{\ell^{\prime} j^{\prime}}^{k} \mathbf{X}_{\ell^{\prime} i} \mathcal{C}_{\ell}\left(\mathbf{X}_{i+j-j^{\prime}}\right) \\
=\lambda^{k} \mathcal{C}_{\ell}\left(\mathbf{E}_{j}^{k}\right) .
\end{gathered}
$$

Therefore, since $\mathbf{X}$ verifies the linear constraint $\mathcal{C}$, the spacetime EOFs are also solutions of: $\mathcal{C}_{\ell}\left(\mathbf{E}_{j}^{k}\right)=0$ for all $j$ and $k$. Here, $\mathcal{C}$ acts only on the spatial dimension of $\mathbf{E}$. The $k^{\text {th }}$ mode $\mathbf{X}^{k}(L \times N)$ can be reconstructed using the definition of Plaut and Vautard (1994). The initial field $\mathbf{X}$ is recovered by summing all the reconstructed components. The latter are also subjected to the constraint $\mathcal{C}$ :

$\mathcal{C}_{\ell}\left(\mathbf{X}_{i}^{k}\right)=0$.

These properties are also valid for all eigenproblems using covariance matrices.

In the simplest case, $\mathcal{C}$ refers to a summation on the various terms of an equation at one spatial point. For instance, let $\Phi_{\ell i}$ $(1 \leq \ell \leq 6)$ be the mass flux at time $i$ through the $\ell^{t h}$ edge of an oceanic cubic box. Then

$\sum_{\ell=1}^{6} \Phi_{\ell i}=0, \quad \forall i$

because of constant mass conservation. In this particular case, $\mathcal{C}$ is just the sum over the channels of $\Phi$. Then, all the resulting components $\Phi^{k}$ satisfy the mass conservation.

This mathematical method, derived from MSSA, works as a constraint that is physically coherent. In order to keep a physical significance, the analyzed space-time field must be suitably chosen before being filtered by the diagnostic tool. An example of the methodology is delineated in Sect. 3.

\subsection{Perturbation of a signal}

In this section, we present a way to interpret dynamically the statistical modes given by the MSSA of an equation. The interpretation is based on an estimate of the first order response an academic signal is perturbed in its phase space.

\subsubsection{Definition of an idealized dynamical system}

We start from an oscillatory signal $S$ with an amplitude $A$ and a period $T$ :

$S=A e^{i(\omega t+\phi)}$

where $\omega=2 \pi / T$.

The variability of $S$ is driven by physical processes (e.g. advection, diffusion, forcing... ). Let us assume that the rate of variation of $S$ can be represented by a sum of tendency terms which also have oscillatory signatures:

$\frac{d S}{d t}=\dot{S} \equiv \sum_{k} a_{k} e^{i \varphi_{k}} e^{i(\omega t+\phi)}$

where $a_{k}$ represents the strength of the $k^{t h}$ tendency and $\varphi_{k}$ its phase relative to the main signal $(S)$. This differential 
equation is assumed to contain the physics driving the variations of $S$. We investigate the effect of perturbations of the right hand side of Eq. (9) on the behavior of $S$.

Figure 1 schematizes the oscillation and the contributions of one and the sum of all tendencies at $t=0$. The oscillation is assumed stationary at the first order and describe here a circle, so the sum of the tendency contributions is naturally tangential to this circle.

\subsubsection{Solving the perturbed system}

All along the cycle we apply a small perturbation of the $\ell^{\text {th }}$ tendency term that slightly increases or decreases the strength of the associated process, which corresponds to the following transformation:

$a_{\ell} \rightarrow a_{\ell}(1+\epsilon) \quad$ with $\quad \epsilon$ small.

In a linearized context, the perturbation may be considered as a small change in the background advection affecting only one advective tendency. The objective is to estimate the impact of this small perturbation. For simplification and without loss of generality, we assume that the phase $\phi$ is zero. Let $S_{\ell}^{\prime}$ be the resulting perturbed signal. Its time derivative is:

$\dot{S}_{\ell}^{\prime}=i \omega S^{\prime}+P_{\ell}$,

where the perturbation function $P$ is added to the derivative of Eq. (8). By continuity, $P_{\ell}=\mathcal{O}(\epsilon)$, because $S_{\ell}^{\prime}$ becomes the unperturbed solution when $\epsilon \rightarrow 0$. Thus we can assume that the system preserves its internal dynamical equilibrium. Therefore, if the amplitude and/or period of the system are perturbed by multiplicative factors (like in Eq. 10), then the amplitude and period of each tendency vary accordingly (see Eq. 9). So we choose to take a perturbation function that is proportional to the perturbed signal $S^{\prime}$, with $p$ the complex proportionality coefficient:

$P_{\ell}=p_{\ell} S_{\ell}^{\prime}$

and with

$P_{\ell}=\epsilon a_{\ell} e^{i \varphi_{\ell}}=p_{\ell} A, \quad$ at $t=0$

we obtain

$\dot{S}_{\ell}^{\prime}=\left(i \omega+\frac{\epsilon a_{\ell}}{A} e^{i \varphi_{\ell}}\right) S_{\ell}^{\prime}$.

We search a solution with the following form, with all subscripts $\ell$ removed for clarity:

$S^{\prime}=A^{\prime} e^{\left(\gamma^{\prime}+i \omega^{\prime}\right) t+\phi^{\prime}}$.

Deriving Eq. (15) and by analogy with Eq. (14), we obtain the two parameters involved in the time evolution of the solution:

$$
\left\{\begin{array}{l}
\gamma^{\prime}=\frac{\epsilon a}{A} \cos \varphi, \\
\omega^{\prime}=\omega+\frac{\epsilon a}{A} \sin \varphi .
\end{array}\right.
$$

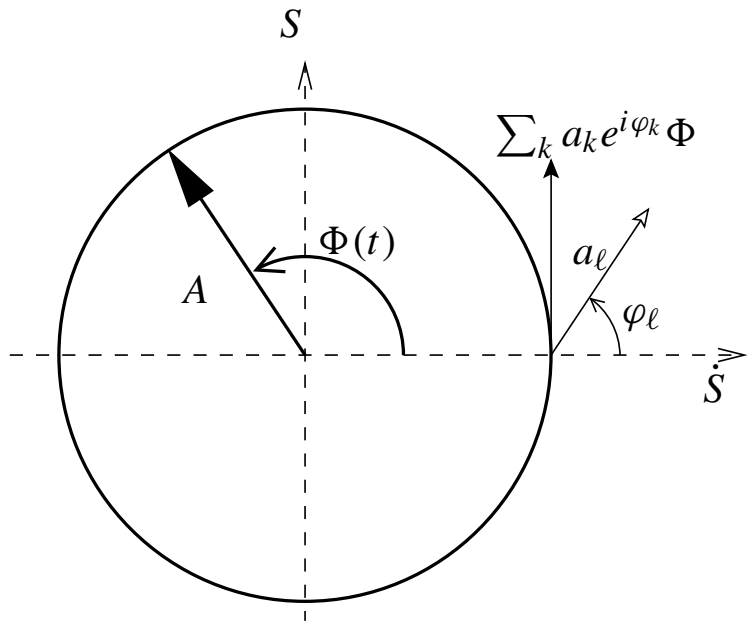

Fig. 1. Cycle of the oscillation $S$ in the phase space. Also shown, the $\ell^{t h}$ tendency $\left(a_{\ell} e^{i \varphi_{\ell}}\right)$ and the total tendency at a particular time.

If the perturbation is applied at $t=0$ and with $\phi=0$ for simplicity, we obtain the two equations:

$\begin{cases}\Re\left(S^{\prime}\right)=A^{\prime} \cos \phi^{\prime} & =A, \\ \Re\left(\dot{S}^{\prime}\right)=A^{\prime}\left(\gamma^{\prime} \omega \cos \phi^{\prime}-\omega^{\prime} \sin \varphi^{\prime}\right) & =\epsilon a \sin \varphi,\end{cases}$

where $\Re$ is the real part of a complex number. We deduce the last two parameters of the solution of Eq. (15):

$$
\left\{\begin{aligned}
A^{\prime} & =\frac{A}{\cos \phi^{\prime}}, \\
\tan \phi^{\prime} & =\frac{\cos \varphi}{\frac{\omega A}{\epsilon a}+\sin \varphi} .
\end{aligned}\right.
$$

These two parameters can induce an initial phase shift and affect the amplitude. A more general case is obtained when the perturbation does not start when the initial signal is maximal. If $\epsilon$ is small, $\phi^{\prime}$ remains close to zero, and $A^{\prime}$ close to $A$. Their effect is small and constant, contrary to the two other parameters $\left(\gamma^{\prime}\right.$ and $\left.\omega^{\prime}\right)$ whose effect on the cycle grows in time until an equilibration is reached due to the weak nonlinearities.

\subsubsection{Impact on the amplitude and frequency}

In general, the signal is not a simple oscillation with constant amplitude and period. Here we evaluate at the first order their respective tendency according to the perturbation parameters $\varphi$ and $\epsilon$.

Figure 2 is an example of perturbed cycle after a time corresponding to the period. By using the expression of $\gamma^{\prime}$ in Eq. (16), we deduce the first order gain in amplitude, as indicated in the figure.

The only term modulating the amplitude of the perturbed cycle is $\gamma^{\prime}$ (see Eq. 16) which represents the growing rate of $S^{\prime}$. It is proportional to the strength of the perturbation and to the radial component $a \cos \varphi$ of the tendency term on which it is applied. 


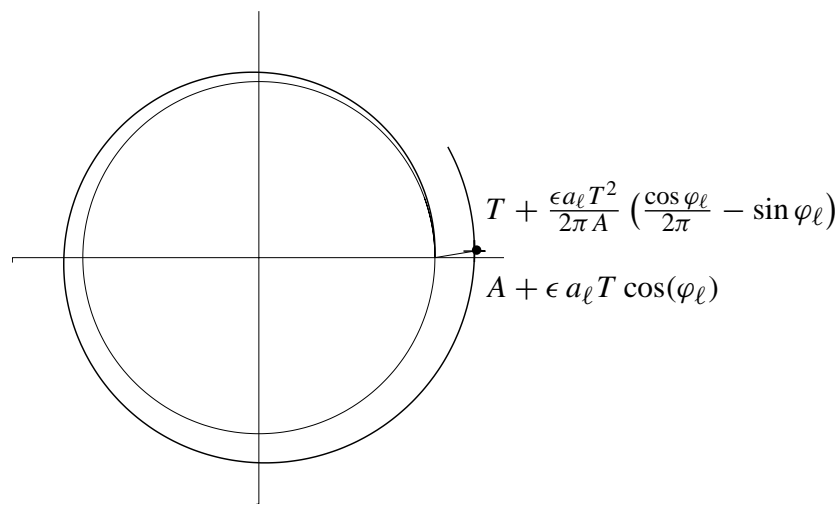

Fig. 2. The perturbed oscillatory regime computed with an idealized numerical model.

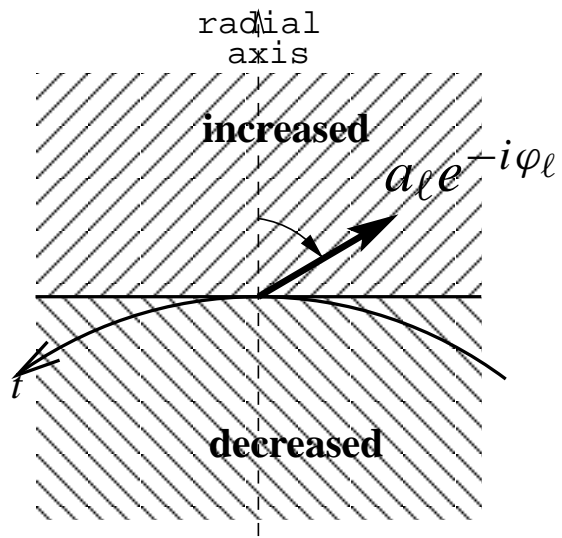

Fig. 3. Regions defining a positive and a negative impact of a tendency term of the amplitude of the cycle: if $\varphi_{\ell}>\pi / 2$ it is a decrease, otherwise it is an increase.

This result is illustrated in Fig. 1: when the tendency is oriented outside of the circle, it basically tends to increase the radius of the cycle. The growth rate of the amplitude is given by:

$\dot{A}=\epsilon a \cos (\varphi)$.

The impact of a perturbed tendency term on the amplitude of the trajectory is illustrated in Fig. 3.

The response of the period of the oscillation to the perturbation is driven by a direct and an indirect effect, which can compete with one another.

The direct effect is found in the expression of the modified frequency of the cycle $\omega^{\prime}$ (see Eq. 16) and is proportional to the tangential component of the tendency term on which the perturbation is applied. When the term is oriented in the same direction as the evolution of the trajectory (counterclockwise on Fig. 4) and is enhanced, the signal goes from one phase to an other in a quicker way, thus the period decreases.

The indirect effect is due to a modulated amplitude of the cycle that can influence the transition time between extrema $T^{\prime}$, defined as the "pseudo period" of the perturbed cycle. In

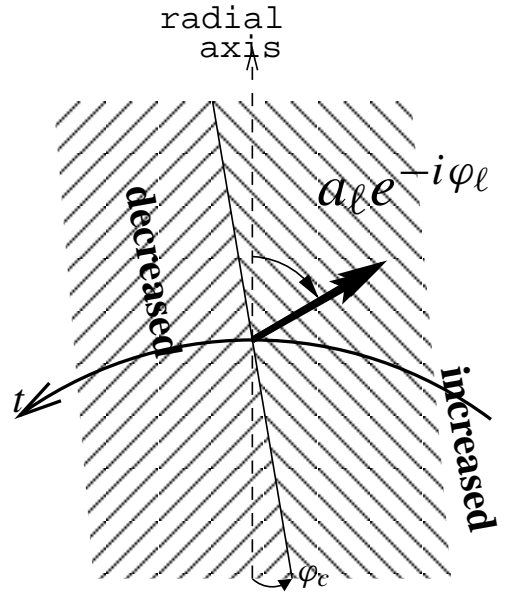

Fig. 4. Same as Fig. 3 but for the period of the cycle. In addition, it is shown that the impact is not purely dependent on the phase $\varphi$ but on its value with respect to $\varphi_{c}$ : if $\varphi_{\ell} \in\left[\varphi_{c}, \pi+\varphi_{c}\right]$ the period is decreased, else it is increased.

order to evaluate it, we compute this time by finding when the cycle reaches a maximum near $t=T$. It is the solution of:

$\dot{S}^{\prime}(t+\delta t)=A^{\prime}\left(\gamma^{\prime}+i \omega^{\prime}\right) e^{\left(\gamma^{\prime}+i \omega^{\prime}\right)(t+\delta t)+\phi^{\prime}}=0$.

The growth rate of the period is defined by $\dot{T}=\delta T / T$ at a particular time $t \simeq T$. We deduce it at the first order from the solution of Eq. (16):

$\dot{T}=\frac{\epsilon a T}{2 \pi A}\left(\frac{\cos \varphi}{2 \pi}-\sin \varphi\right)$.

The corresponding gain in amplitude after a time $t \sim T$ is shown in Fig. 2. The RHS of Eq. (21) contains two term in competition, to control the sign of the derivative $\dot{T}$. When $\varphi$ is larger than a critical value of $\varphi_{c}$, the period decreases. If $\varphi<\varphi_{c}, T$ increases. This is schematized in Fig. 3 where the limit in the phase space between a positive and a negative impact on the period is shown. We find from Eq. (21) the critical angle $\varphi_{c}$ separating the two regions:

$\varphi_{c}=\arctan (1 / 2 \pi) \simeq 9^{\circ}$.

Hence, one need to take to compare $\varphi$ to $\varphi_{c}$ for interpretations in the phase space.

\section{Physical application}

In this part, we apply our theoretical results on a concrete case. The idea is to use a numerical model, to suitably perturb one of its equations in several experiments, and to describe the observed relationship between the characteristics of the perturbation and the way in which the signal is changed.

\subsection{The climate model}

This application focuses on the impact of physical parametrization on the El Niño/Southern Oscillation (ENSO) 
variability patterns. We use a hybrid coupled model of the tropical Pacific and Atlantic oceans. This model is composed of an intermediate complexity ocean model coupled to a simple statistical atmospheric model. This model exhibits spontaneous oscillations in the tropical Pacific which are related to ENSO variability. The objective of this section is illustrate our methodology on the dynamics of this model of intermediate complexity.

The ocean model configuration is that of Zhang et al. (2005). The linear dynamical part is inspired from the modal model (McCreary, 1981). In our formulation, a varying background stratification is used for the vertical modes decomposition (Keenlyside and Kleeman, 2002). Ten modes are used to compute the linear dynamics, and the remaining modes are integrated over two surface layers to diagnose the Ekman dynamics. The first layer is the mixed layer with a depth $H$ (constant in time) computed using a stability criterion and the mean temperatures and salinities from Levitus (1982). The base of the second layer is fixed at depth $\mathrm{H}_{2}$ of $125 \mathrm{~m}$. Non linear terms are integrated over the first two layers to correct the linear dynamics. The thermodynamical model is an anomaly model forced by the dynamical part, and described by the following equation

$$
\begin{aligned}
\frac{\partial T^{\prime}}{\partial t}= & -u^{\prime} \frac{\partial \bar{T}}{\partial x}-\left(\bar{u}+u^{\prime}\right) \frac{\partial T^{\prime}}{\partial x}-v^{\prime} \frac{\partial \bar{T}}{\partial y}-\left(\bar{v}+v^{\prime}\right) \frac{\partial T^{\prime}}{\partial v} \\
& {\left[\left(\bar{w}+w^{\prime}\right) M\left(-\bar{w}-w^{\prime}\right)-\bar{w} M(-\bar{w})\right] \frac{\left(\bar{T}_{e}-\bar{T}\right)}{H} } \\
& -\left(\bar{w}+w^{\prime}\right) M\left(-\bar{w}-w^{\prime}\right) \frac{\left(T_{e}^{\prime}-T^{\prime}\right)}{H}-\alpha T^{\prime} \\
& +\frac{\kappa_{h}}{H} \nabla_{h} \cdot\left(\nabla_{h} T^{\prime}\right)+\frac{2 \kappa_{v}}{H\left(H+H_{2}\right)}\left(T_{e}^{\prime}-T^{\prime}\right)
\end{aligned}
$$

where $T$ and $T_{e}$ refer to the sea surface temperature (SST) and subsurface temperature, $M$ is the Heaviside function, $\alpha$ the damping coefficient for the sea-air heat flux, $\kappa_{h}$ and $\kappa_{v}$ the horizontal and vertical diffusion coefficients. The primes denote anomalies and overbars refer to the climatological fields. The dynamical climatology is computed using a forcing climatology from the National Center of Environmental Prediction (NCEP) (Kalnay et al., 1996). The observed SST climatology is given by Reynolds et al. (2002). The subsurface temperature $T_{e}^{\prime}$ anomalies are derived from the sea surface height anomalies using a statistical relationship, and the climatology $\overline{T_{e}}$ is computed from $20^{\circ} \mathrm{C}$ isotherm depth with an empirical formula (see Zhang et al., 2005). The zonal resolution of the model is of $2^{\circ}$. The meridional resolution ranges from $0.5^{\circ}$ at the equator to $3^{\circ}$ at the meridional boundaries.

The statistical atmospheric model is first built by an independant EOFs analysis of SST and wind stress anomalies. A regression matrix is then computed between the first four PCs of SST and the first two PCs of wind stress. To deduce wind stress from SST anomalies, the model projects wind stres anomalies onto the SST EOFs of the model, projects the resulting SST coefficients onto the regression matrix to compute the wind stress coefficients, and multiplies them by the wind stress EOFs of the model.

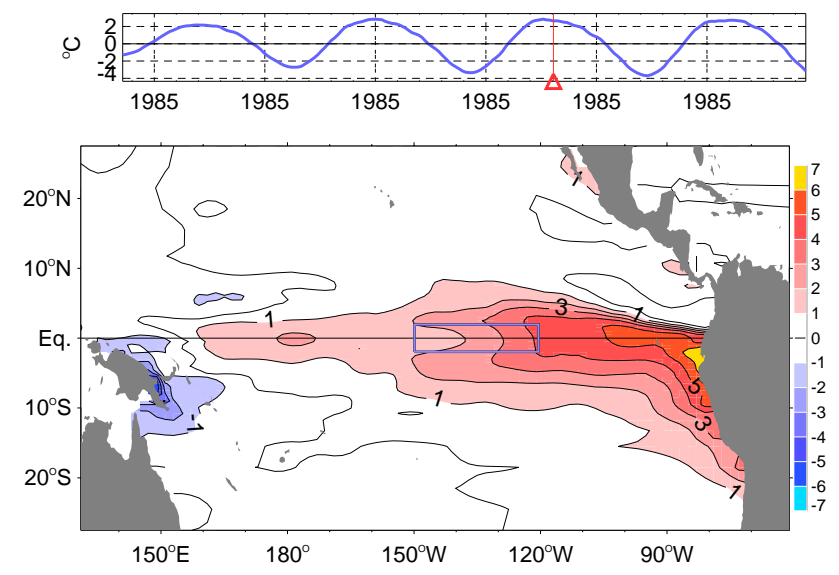

Fig. 5. Averaged SST anomaly in the $\left[150^{\circ} \mathrm{W}: 120^{\circ} \mathrm{W}, 2^{\circ} \mathrm{S}: 2^{\circ} \mathrm{N}\right]$ box along the 19 year simulation (upper panel). Anomaly of the coupled model at a particular time during year 1994 (lower panel), and box limits (in blue).

We performed 919 year simulations (1 control simulation and 8 sensitivity experiments), analyzing only the last 17 years. Figure 5 shows the typical pattern and amplitude of the simulated SST. A central Pacific box $\left[150^{\circ} \mathrm{W}\right.$ : $\left.120^{\circ} \mathrm{W}, 2^{\circ} \mathrm{S}: 2^{\circ} \mathrm{N}\right]$ is used to compute an index characterizing the SST evolution. This same box delimits the region where the perturbation is applied. The variability yields a $\sim 4$ year oscillation with a realistic pattern (Fig. 5).

\subsection{Application to the SST equation}

The SST Equation (23) is used in our sensitivity study for two reasons. First, the SST is generally chosen as the reference variable for all ENSO modelling studies (Stockdale et al., 1998), and second, this equation is one of the three components of the coupled system, so the basic variability will be affected if a perturbation occurs. The filtering process consists first in reducing the number of the degrees of freedom (to 10) by applying a time EOF (T-EOFs) decomposition, before using the MSSA with a window parameter $M=96$ (months). This filter is applied to the equation with its terms grouped in the following way:

$$
\begin{aligned}
\dot{T}^{\prime} & =\bar{u} \cdot T_{x}^{\prime}+u^{\prime} \cdot \bar{T}_{x}+u^{\prime} \cdot T_{x}^{\prime}+\left(u^{\prime} \cdot T_{x}^{\prime}\right)^{\prime}+\left(v \cdot T_{y}\right)^{\prime}+\left(w \cdot T_{z}\right)^{\prime} \\
& + \text { diffusion }+Q_{\text {surf }},
\end{aligned}
$$

where $\left(u^{\prime} . T_{x}^{\prime}\right)^{\prime}$ is the nonlinear zonal advection anomalies, and $Q_{\text {surf }}$ is the air-sea flux. The analysis is performed in the equatorial box defined in Fig. 5. In this region, anomalies are important and the ocean-atmosphere coupling is very efficient (Stockdale et al., 1998). For consistency with Eqs. (8) and (9), the SST itself is introduced in the analysis. A factor ( $\Delta t=1$ month corresponding to the sampling interval) is applied to the tendencies for physical homogeneity and avoid numerical problems. Figure 6 shows how the tendency terms can be noisy, even with regular variability.

Therefore, MSSA appears very useful especially when analyzing more realistic models, and the methodology 


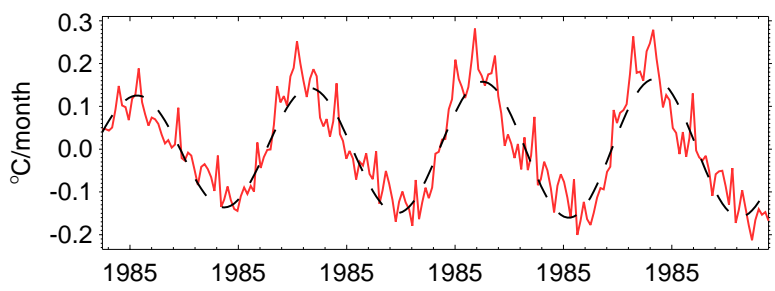

Fig. 6. The initial total tendency ( $\partial T^{\prime} / \partial t$, black) and its first reconstructed MSSA pair of modes (red), averaged over the box as in Fig. 5.

used here has an advantage over the simple regressions of Da Costa and Vautard (1997) for extracting tendencies.

There are two possible coordinate systems to display the phase space:

- A variable of the system and its multiple derivatives, members of a high order equation. We used this representation until now because it is useful when dealing with both physical and dynamical systems.

- Multiple variables of the system, members of a set of low order interrelated equations. In the current statistical context, the modes extracted with MSSA become the variables, (e.g. Da Costa and Vautard (1997) and Wang and Wang (2000), or Hsieh and Tang (1998) in a nonlinear context with more modes). Then, an oscillation can be represented in a space in two dimensions using the associated pair of modes. In the case of a harmonic oscillation, PCs and ST-EOFs are in phase quadrature (Plaut and Vautard, 1994).

We chose the second approach because it uses direct products of the analysis and the axis are homogeneous. For a simple representation of the main cyclic signal (i.e. the SST), only the pair of ST-PCs (for a particular oscillation) is needed. However, in order to distinguish the role of each tendency, ST-EOFs are needed and will provide a signal for each of term. Practically, these ST-EOFs are projected against the T-EOFs to go back from the reduced space (10 d-o-f) to real physical space. The resulting field has a time length equal to those of the ST-EOFs, that is the window parameter length $(M)$. The space-time signal of each tendency is then spatially averaged for the two reconstructed ST-EOFs, and their coordinates $([a, \varphi])$, in the referential defined by radial and orthoradial coordinates of the main signal $([A, \Phi])$, are then estimated and averaged all along time (so along the cycle). We obtain a representation similar to that of Fig. 1.

Using the method on the SST equation, four dominant terms are found and displayed in Fig. 7:

- The air-sea flux $\left[Q_{\text {surf }}\right]$ is the heat dissipation and acts as a damping: it only has a negative radial component as expected because it is linearly opposed to the SST anomaly. However, since this term is strong and oriented in the region where a negative impact on the period is expected (see Fig. 4), it may not act as a simple

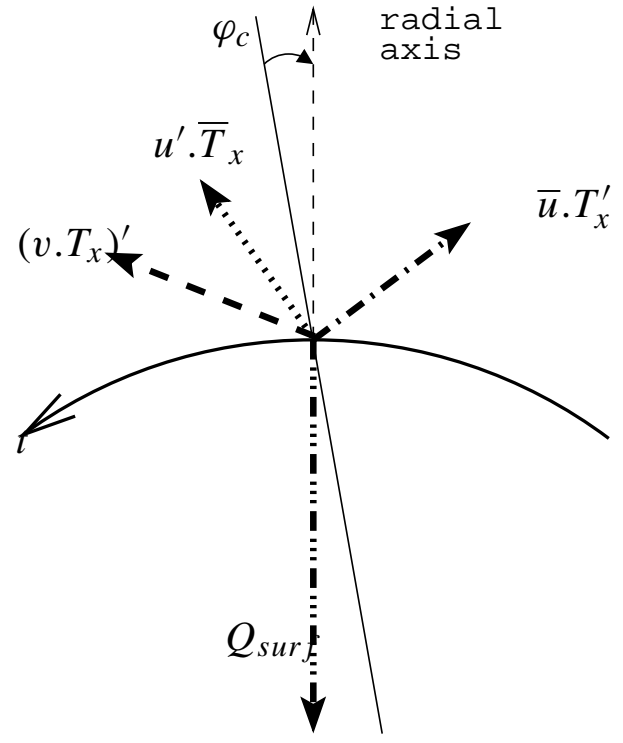

Fig. 7. Schematic view of the dynamic equilibrium resulting from of the analysis of the SST Eq. (24) in the Equatorial box. Only the four dominant terms are displayed. The thin solid line represents the critical axis separating positive and negative contributions to the period (see Fig. 4). This equilibrium is invariant all along the cycle.

damping but also contribute to lower the period of the averaged cycle (so the period of the cycle is decreased). When decreasing SST anomalies in such way, the difference of temperature between extremes is also decreased and it simply takes less time, for example, to transit from an El Niño state to a La Niña state.

- The anomalous zonal advection of background temperature $\left[u^{\prime} \cdot \bar{T}_{x}\right]$ tends to increase the amplitude of the cycle, but also slightly accelerates it. Such precursor and amplificator role for this term due to equatorial waves is well known (Kessler and McPhaden, 1995; Picaut et al., 1997; Boulanger and Menkes, 1999). Basically, during a positive event, the westward zonal current (South Equatorial Current - SEC) is weaker in the center of the basin due to weaker trade winds. Thus, an anomalous eastward current advects hot waters inside the box of Fig. 5 from the west. In addition to the direct effect of winds, a part of this anomalous advection, that is due to waves excited during the previous negative event and reflected on the west coast, acts in an opposite way. This reflected signal is already active when SST is maximal inside the box. Consequently it helps to transit to the next cold event and more generally tends to decrease the period of the cycle.

- The meridional anomalous advection $\left[\left(v . T_{y}\right)^{\prime}\right]$ also has a positive impact on the amplitude and a negative contribution to the period. Since this term is averaged in the $\pm 2^{\circ}$ of latitude band, it is coherent with the "recharge 
oscillator" mechanism proposed by Jin (1997), where the meridional advective term is crucial for the transition between extremes (Battisti, 1988; Yu and Mechoso, 2001). This term combines the variability of equatorial divergence due to changes in the trades winds (positive impact), and the extra-equatorial advection of anomalous SST (also a positive contributor since our model shows important SST anomalies slightly off the equator near the box of Fig. 5 during extremes). In addition, according to the "recharge oscillator" concept, during positive events an increased heat storage along the equator due to anomalous convergence progressively deepens the thermocline and reduces the mean zonal SST gradients. This effect acts against the ocean-atmosphere coupling and helps the system to transit to a negative event (and the period becomes shorter).

- The background zonal advection of anomalous temperature $\left[\bar{u} . T_{x}^{\prime}\right]$ has a positive impact on the amplitude and increases the period. Taking into account $\varphi_{c}$, the impact of this term on the period may be particularly efficient. Therefore, is appears as a "passive term", contributing to the growth of SST anomalies but constantly lagging them and working against the termination of the event (Boulanger and Menkes, 2001). During a positive event in the equatorial box of Fig. 5, the background SEC advects SST anomalies that are maximal east of the box and then increases SST inside. However, during the onset of a positive event, SST anomaly is maximal at the west end of the box, then the SEC slows down the heating inside the box by advective cold anomalies (the period is increased).

- Our analysis of the total vertical advective (not shown here because mainly active eastward to this box) confirms its contribution to the growth of the anomalies as show by McPhaden et al. (1998) and Neelin et al. (1998). First, anomalies of trade winds modulate the divergence in the east and create SST anomalies. Second, subsurface temperature anomalies due to changes in the wind forcing and the thermocline depth are advected toward the surface in the east by the mean upwelling. The box of Fig. 5 is only weakly affected by these processes because it is situated in the center of the basin.

Despite the approximations of the model (especially the atmospheric component), the physical properties deduced by this statistical analysis are coherent with what is known of ENSO dynamics in the literature (cited in this section).

In order to verify the dynamical meaning of these statistical results, a perturbation is artificially applied to these four terms in the SST equation when the model is running. We apply a perturbation to the terms of the SST equation when the model is running to test the stability of the oscillatory properties detected in the model. It consists in increasing the terms with by a constant coefficient $(1+\epsilon)$ in Eq. (10) over the region of Fig. 5 with different values $(0.8,1.2)$, resulting in two experiments for each tendency term. The results are shown

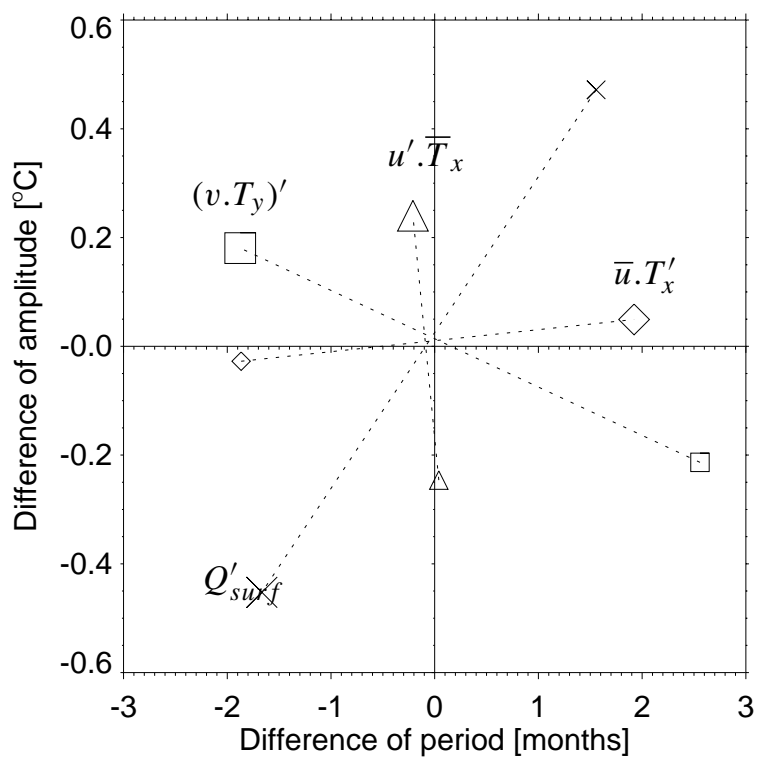

Fig. 8. Results of the perturbation experiments on the $u^{\prime} \cdot \bar{T}_{x}, \bar{u} . T_{x}^{\prime}$, $\left(v . T_{y}\right)^{\prime}$ and $Q_{\text {surf }}$ terms: impact on the amplitude (a) and on the period (b). The differences have been evaluated after four complete cycles.

in Fig. 8. We find that the system responds as predicted by the statistical analyses. For example, the perturbation of the anomalous meridional advective term $\left[\left(v . T_{y}\right)^{\prime}\right]$ creates a cycle with a shorter period ( -1.9 month) and greater amplitude $\left(\sim+0.2^{\circ} \mathrm{C}\right)$. A similar comparison is valid at the first order for the other terms at work. Therefore, the statistical indicators can be interpreted as dynamical indicators.

The two zonal advective terms, $u^{\prime} \cdot \bar{T}_{x}$ and $\bar{u} . T_{x}^{\prime}$, have their respective period and amplitude contribution slightly underestimated. This can be explained by taking into account the effect of the second pair of modes (oscillation). Figure 5 shows that the SST oscillation is not perfectly sinusoidal, because of nonlinear effects. MSSA reacts to these nonlinearities by introducing weak secondary pair of modes, that represent the same dynamical mode as before. Compared with the first one, this secondary oscillation shows a positive contribution to the period for the $u^{\prime} \cdot \bar{T}_{x}$ term and a negative contribution to the amplitude for the $\bar{u} . T_{x}^{\prime}$ term. These differences explain the slight discrepancies between the statistical estimations and the numerical results. This remark shows that, even if recent developments seem able to merge linear modes in a nonlinear one (Hsieh, 2004), linear decompositions may be still useful to diagnose the basic dynamics.

More general conclusions are now possible. For example, if a change in the internal parameters or the external forcing of the model slightly increases the mean zonal current in the studied box, the period of the oscillation will decrease due to $\bar{u} . T_{x}^{\prime}$. This can be partly compensated by a change in the mean meridional and vertical currents. This can modify will 
be also affected, and the period especially through the $\bar{v} \cdot T_{y}^{\prime}$ term included in $\left(v \cdot T_{y}\right)^{\prime}$.

The quantitative responses in amplitude and period to a local perturbation for a particular oscillation are not the same. An oscillation is identified by its spatio-temporal structure and a particular period. Thus, if period of an oscillation is locally affected it must also be globally affected since the period is the same everywhere. However, nothing forces the oscillation to globally adjust itself to a local change in the amplitude. Then the local amplitude may be more easily affected than the period. Such constraint in the period induces a constant phase lag between the local signal and the oscillation elsewhere, which can be viewed as phase locking. A shortening of the period should be typically associated with a local positive lag (advance) with respect to the global signal.

\subsection{More complex cases}

In more realistic configurations, it is expected to have perturbations on variables in space and even in time, affecting multiple tendencies at the same time. The solution is to stay at the first order, treat each tendency separately and then combine them. In other words, it consists in computing the perturbation term $\epsilon a_{\ell} e^{i \varphi}$ for each affected tendency and sum all these vectors: the MSSA will provide the distribution of $a$ and $\varphi$, and the expected change in the mean state due to the perturbation (normalized to this mean state) the distribution of $\epsilon$. Finally $\varphi$ is deduced from the integrated perturbation impact:

$\epsilon a e^{i \varphi}=\sum_{\ell} \sum_{p} \int \epsilon_{p} a_{\ell} e^{i \varphi_{\ell}} d s \quad / \int d s$

where $p$ is the perturbation index (for example associated to the change in background zonal current or in background zonal gradient of SST), $\ell$ is the tendency index, $d s$ the infinitesimal surface element integrated over the region of interest. So, like before, $\varphi$ and $a$ give the key to predict how the system will change in terms of amplitude and period.

\section{Conclusions}

In this paper, we propose and validate a statistical methodology that give access to dynamical information about a quasilinear variability. The MSSA is applied to a closed equation, representative of the studied phenomenon. All the resulting modes verify the equation, and the impact of their tendencies on the period and the amplitude of the variability may be estimated using particular diagnostics. The methodology tested on a hybrid coupled model of ENSO to obtain the sensitivity of the cyclic features to perturbations in the physical parameterizations of the model.

A simplified oscillatory model of one component of a system can be built using our approach. The analysis gives a first order approximation of the effect of suppressing a particular (typically small) term on the mean amplitude and period of the variability. For example, it expected that removing two terms that are approximately opposed in the phase space representation will not change variability, whereas the physics will be different. Thus, our approach gives a synthetic information about the internal dynamics of the model.

In this way, the information obtained from a given model may used to predict the first order changes in the variability in response to a perturbation of the system. Such perturbation are typically due to a change in the internal parameters (including the specified background for an anomaly model) or the external forcing of the model. Raynaud (2000) used this approach to compare the ENSO-like variability of three simulations of coupled ocean general circulation model, differing only the formulation of the lateral ocean diffusion. One part of the differences were explained by indirect effects through subtle modifications of the background state (slow adjustments of the model). The other part of the differences was due to direct effects of the diffusion terms on the variability. Therefore, our statistical diagnostics avoid making too many cpu expensive sensitivity studies, or at least to help planning them.

The synthetic oscillatory model given by our methodology may be built for example to compare the dynamics of two different regions of same model (for example the equatorial variability of the Atlantic and Pacific oceans), or different epochs (last glacial maximum, global warming state). The oscillatory model would be also useful for comparisons between different models. For example, such comparison may concern two complex models built in a different way but supposed to simulate the same variability. A term-to-term comparison of the physical tendencies in the phase space (see in Sect. 3.2) would help to explain the differences in the variability of the two models. This approach is also interesting for comparing complex and simple models. The analysis of complex model could give the qualitative and quantitative role of the terms that were neglected in a simple model. The comparison would help to make the link in terms of internal dynamics between a complex "realistic" model (where advanced statistical methods help to filter huge amount of data) and a more understandable simple "toy" model.

Due to weak nonlinearities in a physical system and the presence of a noisy forcing or a strong seasonal cycle, the variability is generally represented by the sum of a few modes. With our methodology, we can linearly combine the identified modes to obtain the time variations of the system. Many climate phenomena (especially involving the ocean) yield dominant modes in the frequency domain (Bjerknes, 1964; White and Peterson, 1996; McPhaden et al., 1998). Thus, our methodology is appropriate for the study of their weakly nonlinear variability.

However, it should be kept in mind that, like with any statistical tool, an a priori knowledge of the dynamics is necessary before processing so that the methodology is used in a more consistent way: the methodology just renders the path between dynamics and statistics easier and useful.

Acknowledgements. We gratefully thank M. Ghil for his fruitful comments on a preliminary version of this paper. We also thank the 
anonymous reviewer for his comments that helped to improve this paper.

Edited by: A. Tsonis

Reviewed by: one referee

\section{References}

Allen, M. R. and Smith, L. A.: Monte Carlo SSA: Detecting irregular oscillations in the presence of colored Noise., J. Clim., 9, 3373-3404, 1996.

Battisti, D. S.: The dynamics and thermodynamics of a warm event in a coupled atmosphere/ocean model, J. Atmos. Sci., 45, 28892919, 1988.

Bjerknes, J.: Atlantic air-sea interaction, Adv. Geophys., 10, 1-82, 1964.

Boulanger, J.-P. and Menkes, C.: Long equatorial wave reflection in the Pacific Ocean from TOPEX/POSEIDON data during the 1992-1998, Clim. Dyn., 15, 205-225, 1999.

Boulanger, J.-P. and Menkes, C.: The Trident Pacific model. Part 2: The thermodynamical model and the role of long equatorial waves during the TOPEX/POSEIDON period, Clim. Dyn., 17, 175 - 186, 2001.

Broomhead, D. S. and King, G. P.: Extracting qualitative dynamics from experimental data, Phys. D, 20, 217-236, 1986.

Da Costa, E. and Vautard, R.: A qualitative realistic low-order model of the extratropical low-frequency variability built from long record of potential vorticity, J. Atmos. Sci., 54, 1064-108, 1997.

Elsner, J. B. and Tsonis, A. A.: Do bidecadal oscillations exist in the global temperature record?, Nature, 551-553, 1991.

Elsner, J. B. and Tsonis, A. A.: Singular Spectrum Analysis: A New Tool In Time Series Analysis, Plenum Press, New York, 1996.

Ghil, M.: Natural Climate Variability, in Encyclopedia of Global Environmental Change. The Earth System: Physical and Chemical Dimensions of Global Environmental Change, John Wiley \& Sons, Ltd, Chichester, 1, 544-549, 2002.

Ghil, M. and Robertson, A. W.: Solving problems with GCMs: General Circulation Models and their role in the climate modeling hierarchy, in: General Circulation Models Development: Past, Present and Future, edited by: Randall, R., Academic Press, San Diego, 285-325, 2000.

Ghil, M., R.Allen, M., Dettinger, M. D., Ide, K., Kondrashov, D., Mann, M. E., Robertson, A. W., Saunders, A., Tian, Y., Varadi, F., and Yiou, P.: Advanced spectral methods for climatic time series, Rev. Geophys., 40, 3-11-3-13, 2002.

Golyandina, N., Nekrutkin, V. V., and Zhigliavskii, A. A.: Analysis of time series structure: SSA and related techniques, Chapman \& Hall/CRC, Boca Raton, Fla., 2001.

Hsieh, W. W.: Nonlinear multivariate and time series analysis by neural network methods, Rev. Geophys., 42, RG1003, 2004.

Hsieh, W. W. and Tang, B.: Applying neural network models to prediction and data analysis in meteorology and oceanography, Bull. Am. Meteorol. Soc., 79, 1855-1870, 1998.

Jin, F.-F.: An equatorial ocean recharge paradigm for ENSO. Part I: Conceptual model, J. Atmos. Sci., 54, 811-829, 1997.

Kalnay, E., Kanamitsu, M., Kistler, R., Collins, W., Deaven, D., Iredell, L. G. M., Saha, S., White, G., Woollen, J., Zhu, Y., Leetmaa, A., Reynolds, R., Chelliah, M., Ebisuzaki, W., W.Higgins, Janowiak, J., Mo, K. C., Ropelewski, C., Wang, J., Jenne, R., and
Joseph, D.: The NCEP/NCAR 40-Year Reanalysis Project, Bull. Am. Meteorol. Soc., 77, 437-471, 1996.

Keenlyside, N. and Kleeman, R.: On the annual cycle of the zonal currents in the equatorial Pacific, J. Geophys. Res., 107, 2002.

Kessler, W. S. and McPhaden, M. J.: Oceanic equatorial waves and the 1991-1993 El Niño, J. Clim., 8, 1757-1774, 1995.

Levitus, S.: Climatological atlas of the world ocean, Tech. rep., NOAA Prof. Paper 13, 1982.

McCreary, J. P.: A linear stratified ocean model of the equatorial undercurrent, Phil. Trans. R. Soc. London A, 298, 603-635, 1981.

McPhaden, M. J., Busalacchi, A. J., Cheney, R., Donguy, J.R., Gage, K. S., Halpern, D., Ji, M., Julian, P., Meyers, G., Mitchum, G., Niiler, P., Picaut, J., Reynolds, R. W., Smith, N., and Takeushi, K.: The Tropical Ocean-Global Atmosphere observing systeme: A decade of progress, J. Geophys. Res., 103, 14 169-14 240, 1998.

Neelin, J. D., Battisti, D. S., Hirst, A. C., Jin, F.-F., Wakata, Y., Yamagata, T., and Zebiak, S. E.: ENSO theory, J. Geophys. Res., 103, 14 261-14 290, 1998.

Picaut, J., Masia, F., and du Penhoat, Y.: An advective-reflective conceptual model for the oscillatory nature of ENSO, Science, 277, 663-666, 1997.

Plaut, G. and Vautard, R.: Spells of Low-Frequency Oscillations and Weather Regimes in the Northern Hemisphere, J. Atmos. Sci., 2, 210-236, 1994.

Preisendorfer, R.: Principal component analysis in meteorology and oceanography, Elsevier Sci., 426 pp., 1988.

Raynaud, S.: Impact de la diffusion latéral océanique sur la variability interannuelle du Pacific tropical dans un modèle couplé global de circulation générale, Ph.D. thesis, Université de Bretagne Occidentale, Brest, France, 2000.

Reynolds, R. W., Rayner, N. A., Smith, T. M., Stokes, D. C., and Wang, W.: An Improved In Situ and Satellite SST Analysis for Climate, J. Clim., 15, 1609-1625, 2002.

Stockdale, T. N., T. Anderson, D. L., Alves, J. O. S., and Balmaseda, M. A.: Global seasonal rainfall forecasts using a coupled ocean-atmosphere model, Nature, 392, 370-373, 1998.

Vautard, R. and Ghil, M.: Singular spectrum analysis in nonlinear dynamics, with application to paleoclimatic time series, Physica D, 35, 395-424, 1989.

von Storch, H. and Zwiers, F. W.: Statistical Analysis in Climate Research, Cambridge Univ. Press, Cambridge, 2001.

Wang, R. and Wang, B.: Phase space representation and characteristics of El Niño La Niña, J. Atmos. Sci., 57, 3315-3333, 2000.

White, W. B. and Peterson, R. G.: An Antarctic circumpolar wave in surface pressure, wind, temperature and sea-ice extent, Nature, 380, 699-702, 1996.

Wilks, D.: Statistical Methods in the Atmospheric Sciences, Cornell University, Ithaca, New York, 1995.

Yiou, P., Sornette, D., and Ghil, M.: Data-adaptive wavelets and multi-scale singular-spectrum analysis, Physica D, 142, 254 290, 2000.

Yu, J. and Mechoso, C. R.: A Coupled Atmosphere-Ocean GCM Study of the ENSO Cycle., J. Clim., 14, 2329-2350, 2001.

Zhang, R.-H., Kleeman, R., Zebiak, S., Keenlyside, N., and Raynaud, S.: An empirical parametrization of subsurface entrainment temperature for improved SST simulations in an intermediate ocean model, J. Clim., 8, 350-371, 2005. 